\title{
Research on the Present Situation of Public Sports Products Supply in Rural Areas
}

\author{
Ying Yang \\ College of Physical Education \\ Sichuan Agricultural University \\ Ya'an, China 625014
}

\author{
Liang Zhai \\ College of Physical Education \\ Sichuan Agricultural University \\ Ya'an, China 625014
}

\begin{abstract}
This article aims at investigating the facilities, types, evaluation conditions and sources of funding of public sports products in rural areas. The results show that there is a single source of funding for public sports products supply in rural areas in Sichuan Province, Lack of facilities, there is a contradiction between supply and demand, which can't meet the needs of farmers; sports institution is not perfect. It should considere the lack of organized and guided sports activities and other issues. Here are suggestions for related issues, to provide reference for improving the supply of public goods in rural areas in Sichuan Province.
\end{abstract}

Keywords-Sichuan province; countryside; sports public product; supply

\section{INTRODUCTION}

While thoroughly implementing the spirit of the 18th CPC National Congress and the 3rd Plenary Session of the 18th CPC Central Committee in 2014, the Central Rural Work Conference proposed that the issue of resolving "agriculture, rural areas and farmers" should be taken as the most important task of the entire party and that the policy of The policy of agricultural rich peasants. Public goods in rural areas are products or services that are non-exclusive and non-competitive. The rural sports industry is an important part of rural public issues. With the improvement of people's life, farmers' demand for sports public goods is growing. However, the supply of public sports products in rural areas is far behind the growth of demand, which becomes the main drawback that restricts the development of rural sports. Sichuan Province has 48036 villages under its jurisdiction, including plains, hills, basin mountainous areas, mountains in southwestern Sichuan and Plateau Mountains. In order to improve the efficiency of supply of public sports products in rural areas of Sichuan Province and achieve a balanced development between supply and demand, this research aims to promote the supply of sports equipment and services in rural areas in Sichuan province, and lay the foundation of new rural construction.

\section{OBJECTS AND METHODS}

\section{A. Research Object}

In this paper, rural residents and village cadres in 123 counties (cities and districts) of 21 cities (prefectures) in Sichuan Province were selected as research objects.

\section{B. Research Methods}

1) Documentation: According to the purpose and content of the research, the documents and materials related to the supply of public sports products in rural areas were consulted, retrieved, analyzed and utilized.

2) Questionnaire: Through the questionnaire on the residents of 123 counties (cities, districts) in 21 cities (prefectures) in Sichuan Province, we know the supply of public sports products in rural areas. A total of 3000 questionnaires were distributed, 2954 questionnaires were recovered, 2905 valid questionnaires, the percentage of usable data of is $96.83 \%$.

3) Interview: An interview was conducted with the village cadres in randomly selected villages to conduct research targeted on the equipment, types, evaluations of rural sports facilities in Sichuan Province and the sources of sports funds so as to make a comprehensive study on the current development of rural sports in Sichuan Province Directly understand.

4) Data Statistics: All data are read, calculated and dealt with by MS EXCEL statistical software.

\section{RESEARCH FINDINGS AND ANALYSIS}

\section{A. Status Quo of Physical Supply of Physical Public Goods in Sichuan}

1) Rural sports public facilities and equipment supply of Sichuan: Sports venues and facilities are the material basis for people to participate in sports and are the conditions and carriers for the formation of rural fitness and culture. They are the guarantee for the nationwide fitness. In the investigation of "Equipment in Sports Public Places", 92 out of 123 village counties (cities and districts) have facilities for public sports and public facilities, while $8 \%$ do not. This 
fully shows that with the state's emphasis on rural sports, the corresponding policy support and tilt, the construction of rural public sports facilities has been basically achieved. However, during the survey, at present, the facilities of rural sports venues are mainly concentrated in village and township schools, accounting for $60 \%$ of the total. In order not to affect teaching and manageability, the venues and facilities in schools are generally not open to the public. It also affects the enthusiasm of local villagers in physical activity. Diverse kinds of sports public facilities are suitable for the peasants' sports activities, which can effectively stimulate the initiative and consciousness of the villagers to participate in the sports activities, cultivate their awareness of physical activity of "living well-being and healthy body", and increasing physical education Exercise frequency and frequency, so as to activate their massive leisure life, improve their health level, establish a good social fashion.

2) Analysis on situation of rural areas' public facilities and sports facilities in Sichuan: The scale and level of the development of sports venues are one of the most important symbols of a country's level of economic development and social civilization. Sports facilities are the material basis for the development of sports and are one of the key factors for popularizing mass sports and raising the level of sports. According to the survey on "Which sports venues or equipment are available in your village (multiple choices)", among the choices, the coverage of "small playgrounds", "basketball courts" and "table tennis courts" chosen by residents ranked the top three Bit, respectively $63.35 \%$, $60.59 \%$, $50.10 \%$, while in terms of the villagers to participate in sports activities from their point of view, they put "walking" "running" "mountain" in an important position, these programs are characterized by: do not need special venues and facilities, the project is simple and easy, with environmental characteristics, reflecting the objective reality that facilities in rural areas in Sichuan is still relatively backward, but also rural residents do not understand the choice of fitness results. Living in the fourth, fifth, sixth and seventh venues are "chess room", "others" "integrated style room" and "single parallel bars", the proportion is $36.42 \%, 30.12 \%, 21.29 \%$ and $18.34 \%$ respectively, while the billiards room, fitness Room and other high-end sports venues with lower coverage of equipment, came in the last two, were $6.16 \%$ and $6.56 \%$, showing a clear type of distribution and imbalance, leading to various villages carrying out physical activity Limited, and lacking in sports-specific projects in all regions.

3) Sports equipment mainly used in in rural areas in Sichuan Province: The results of the survey on "the facilities (which can be used in a number of ways) where the primary activities of your village for the use of sports activities are conducted" show that the majority of villagers carrying out sports activities are mainly located in "school districts", accounting for $56.48 \%$ of the total. In 2014 , the sixth national stadium census data communique covers an area of 1,056 million square meters of education system, accounting for $53.01 \%$ of the total. However, the proportion of schools in rural sports venues is higher, while in western Sichuan, the situation is more prominent. School venues are non-public sports venues, which will to some extent restrict the effective implementation of rural physical exercise activities. "Public sports venues" ranked second, accounting for $30.04 \%$, indicating that with the prosperity of the country and people's living standards, the construction of rural public sports venues is getting better and better. "Township-owned sports venues" accounted for 9.56\%, "the rest of the area" the least, accounting for only $3.89 \%$. The availability of facilities for sports facilities is an important measurement of the supply of public goods in rural areas. From the survey data that the number of public sports venues in Sichuan Province is still insufficient, which directly affects the local rural areas to carry out a series of effective sports activities.

4) Satisfaction rate of rural residents in Sichuan Province with supply of facilities in public sports stadiums: An important indicator to measure the supply of public sports products in rural areas is the evaluation of the satisfaction of farmers with sports public goods. In the survey of residents' satisfaction with the supply of facilities in public sports venues, the villagers said they were "very satisfied" with $12 \%$ of the facilities in the stadium and $25 \%$ with "more satisfied", and more think that the facilities of the venues There are many areas which need to be improved, accounting for $50 \%$ of the "average" and $13 \%$ of the peasants who are "very dissatisfied" with the existing site facilities. They think that the site seriously affects and restricts their enthusiasm for physical exercise it's the data above that shows that the supply of sports facilities in rural areas of Sichuan Province cannot meet the needs of peasants themselves. The contradiction between supply and demand is also one of the important factors restricting the development of rural sports.

5) Rural residents in Sichuan Province, the source of funding for rural sports survey: In the survey on the source of rural sports funding, peasants think that the main sources of sports funding include the village financial expenditures, superior financial allocation, social, enterprise and individual donations, and other six aspects. Among them, the proportion of higher-level financial appropriation and social, enterprise and individual donations was relatively large, accounting for $27.00 \%$ and $24.75 \%$ respectively. Diverse source and policy support are reliable guarantees for sports funding, and adequate funding for rural areas to carry out various physical activities of the material premise. Among the villages surveyed, the source of sports funding shows a diversified trend, and the proportions of different factors show a small difference. On the one hand, it reflects the regional diversity among the villages. On the other hand, the financial expenditure of the village accounts for the proportion of view, but also reflects the side of the village 
on the issue of sports funding policy support needs to be strengthened. Only with the support of financial policies, rural sports can really have long-term and vigorous potential for development.

\section{B. Survey on Rural Residents to Non-physical Sports Public Products Supply Status Quo in Sichuan Province}

1) Investigation of the current situation of rural sports activities in Sichuan province: In the relevant survey on "the status quo of the local rural sports activities", $13 \%$ of the villages carried out some regular sports activities and $80 \%$ did "seldom carry out", which fully shows that all sports and cultural activities in rural areas in Datong are less developed, but also failed to meet the needs of the new rural culture. The real situation of sports activities held in the rural areas of Sichuan Province every year is that the number of sports activities held is very small, the number of sports activities is single, the time for carrying out activities is unreasonable, the number of people participating in the activities is very limited, and the total number of participating peasants is not reached at all Participate in rural sports activities.

2) Investigation of rural PE instructors and sports organizations in Sichuan Province: Sports organizations are social entities that realize the common goal of sports and are organized in accordance with certain structural forms and carry out sports activities according to specific rules, which guarantee the sustainable and effective sports activities. Social sports instructor is to guide the mass sports activities in the skills to teach, exercise guidance and organization and management personnel. In the survey of "whether there are sports organizations and sports instructors in the region", only $16.04 \%$ of the sports participation in rural areas of Sichuan is organized at present. The activities are mainly carried out spontaneously or by companions, with $83.96 \%$ the sports organizations that belong to peasants themselves have directly led to very few organized sports activities in rural areas, which reflected the unsound management of sports organizations in rural areas and seriously hindered the development of rural sports activities. $84.69 \%$ of the surveyed social sports instructors in rural areas do not have social sports instructors, so they cannot conduct targeted scientific guidance on rural residents' physical exercise, and have difficulty in popularizing mass sports activities. The rural residents participating in physical exercise are also mostly walking, running, climbing and other mainly. "Do not know how to exercise" is also an objective factor restricting the rural residents in Sichuan from participating in physical activities.

\section{CONCLUSION}

\section{A. Increase Government Support, Vigorously Develop the Rural Economy}

Economy is the key factor of restricting sports consumption. Sichuan must take the development of the western region as an opportunity to increase regional development by means of two aid policies. First and foremost, establish the body color public welfare fund distribution support policies, not only to increase provincial sports lottery public welfare fund to grassroots support force, but also to adjust provinces and municipal sports departments to help the county and township policies. Second, we should establish a horizontal economic cooperation and counterpart assistance mechanism between the eastern and western regions and regions vigorously tap the special economic and competitive industries in various regions and create a new situation of mutual support and common development.

\section{B. Optimize the Policy Environment for Rural Sports Development to Improve Rural Sports Management System}

The establishment of the party and government leaders attaches importance to the sports department-led, supported by the relevant departments, extensive participation of all sectors of the community operating mechanism. We will improve the multi-level peasant sports association at the county, township and village levels and establish a network of rural sports organizations with the town as the leader and the village committee as the foundation and the peasant body association as the link. Increase propaganda efforts to promote sports knowledge to farmers, to understand the scientific method of exercise, and gradually develop their awareness of physical exercise.

\section{Strengthen the Site Facilities of "Revitalize the Use of" and Construction Measures}

Site facilities are the main reason for restricting rural development. The plain in Sichuan Province must learn from the experience of rural sports construction in developed areas to activate and make use of all available resources on the one hand and to further improve the mechanism of sports construction on the other. As a whole, a governmentsupported and socially supported fund put the pattern into the main body with the mode of "striving for higher level support, combining with input at the same level, and using supportive and counterpart support".

\section{Implement Rural School Sports as the Leading Regional Sports Coordination Strategy}

The Construction of rural sports must realize the priority development strategy of school physical education. Plain area of Sichuan must strengthen the construction of school sports facilities, holidays, free of charge to the community, preferential open; emphasis on physical education teachers and local horizontal linkages, organize social sports instructors and sports backbone training class work to achieve resource sharing.

\section{E. Establish and Improve the Rural Sports Competition System}

Sichuan Province, Municipal Sports Bureau and other competent departments must establish and improve the sports competition system, sports departments in line with the principle of small, diverse, civilized and thrifty, and 
actively carry out the level of economic development and agricultural production in Sichuan adapted to the mass sports activities, stimulate farmers to participate in physical activity enthusiasm.

\section{REFERENCES}

[1] Chang Yi-chen, Zhong Quan-hong. Bottlenecks and countermeasures in the development of sports public service in Northwest rural areas [J] .Gansu Social Sciences, 2013 (3): 199-201.

[2] Tian Kaiyou. Macro-control laws and regulations on insufficient supply of public sports products in rural areas [J]. Theories and Practice of Finance and Economics, 2015, (36) 198: 137-141.

[3] Zhu Han Yi. Discussion on Public Sports Products Supply in Rural Areas [J]. P. Culture, 2007, (7): 14-16.

[4] Zheng Li. Research on the Cooperative Governance Paths of Rural Public Sports Service Supply Reform [J] .Journal of Shenyang Institute of Physical Education, 2016 (6): 19-23.

[5] Zhong Quanhong. Governance of Public Goods Supply in Western Rural Areas from the Perspective of Urbanization [J] .Gansu Social Sciences, 2014 (2) 214-2017-2017.

[6] Wang Meng Yang. Government Public Sports Service Satisfaction Performance Evaluation Index Construction - A Case Study of Shanghai [J]. Sports Science, 2013 (10): 63-70. 